\title{
Systematic early obstetrical assistance at calving: I. Effects on dairy calf stillbirth, vigor, and passive immunity transfer
}

\author{
M. Villettaz Robichaud, ${ }^{* 1}$ D. L. Pearl, ${ }^{*}$ S. M. Godden,† S. J. LeBlanc, ${ }^{*}$ and D. B. Haley ${ }^{* 1}$ \\ *Department of Population Medicine, Ontario Veterinary College, University of Guelph, Guelph, ON, N1G 2W1, Canada \\ †Department of Veterinary Population Medicine, University of Minnesota, St. Paul 55108
}

\begin{abstract}
A critical time for dairy cattle is the perinatal period. Good calving management is critical to reduce periparturient losses and ensure the health of the offspring. Generally, it has been recommended that cows be allowed to calve unassisted when possible, but very few studies have been published that support or refute this general guideline. To investigate the effect of early assistance, a clinical trial enrolled 257 Holstein cows that were observed through the second stage of calving and assigned randomly to 1 of 2 calving interventions: not assisted (NA) or early assistance (EA) during the second stage of parturition. Early assistance was given 15 min after the first sight of both front hooves of the calf and done using only human force. After calving, the animals were classified into 4 actual calving intervention groups: too quick to be assisted (TQ), NA, EA, and late assistance (LA; for cows in the NA group that did not calve unassisted within the $1 \mathrm{~h}$ maximum time frame allowed). Giving early assistance to cows during calving as a routine management practice (assigned intervention) did not negatively influence calves' stillbirth risk, vigor at birth, or transfer of passive immunity. Calves in the LA intervention group had significantly greater odds of stillbirth than calves in the NA and EA groups, respectively. Calves in the LA group also had significantly worse vigor at birth than calves in the TQ, NA, or EA groups. Early assistance given at calving to cows that did not present signs of calving difficulties did not adversely affect calves' likelihood of being stillborn, vigor at birth, or transfer of passive immunity. Key words: calving assistance, stillbirth, calf vigor
\end{abstract}

\section{INTRODUCTION}

Parturition is perhaps the most important moment in the life cycle of dairy cows. The goal of good calving

Received March 24, 2016.

Accepted September 17, 2016.

${ }^{1}$ Corresponding authors: marianne.villettaz@gmail.com and dhaley@uoguelph.ca management is not only to allow the dam to initiate her lactation in good health but to also obtain a live and healthy calf that will become a profitable replacement milking cow, if female, or a valuable beef animal, if male. Over $50 \%$ of primiparous and close to $30 \%$ of multiparous dairy cows may need assistance during calving (Lombard et al., 2007). Internationally, the risk of dystocia has been found to be around $5 \%$, but higher risks have been documented $(13.7 \%)$ in the United States (Mee, 2008a). This discrepancy in risk may be explained by the different criteria used for providing assisted calving, which vary from routine assistance, to intervention after a certain time, to obstetrical malpresentations (Mee, 2008a). Overall, there is no consistent scoring system for dystocia (calving ease), but 3- to 5 -point ordinal scales are generally used to score the degree of difficulty or intervention in calving (Mee, 2008a).

Dystocia has a wide range of significant effects on calves, and these effects have been widely researched (Mee, 2008b; Arnott et al., 2012). Producers have reported that $60 \%$ of their calf loses happened at birth (Spicer et al., 1994). Calves born from mild dystocia have 2.3 times (95\% CI, 1.6-3.3) greater odds of being stillborn than calves born from unassisted calvings, and those born from severe dystocia have 15.4 times (95\% CI, 8.5- 27.8) greater odds (Lombard et al., 2007; Tenhagen et al., 2007). The odds of stillbirth also increase greatly if the second stage of labor (from entrance of the calf into the pelvis until full delivery) is longer than $2 \mathrm{~h}$ (Gundelach, et al., 2009). As well, difficult calvings have been found to negatively affect calves' vigor at birth. Barrier et al. (2012) found that calves assisted during birth were less vigorous than calves born unassisted, when vigor was assessed mainly by early behavior related to standing. Similarly, Murray (2014), using a combination of reflexes and physiological indicators of vigor, found that calves born from easy and hard pulls had significantly poorer vigor than calves born from unassisted calvings. Time to sternal recumbency was found to be a reliable and practical indicator of a calf's likelihood of survival to $7 \mathrm{~d}$ of age; Schuijt and Taverne (1994) found that calves attaining sternal re- 
cumbency 16 min or more after birth were significantly more likely to be nonviable, and that calves born using forced extraction (force of 2 people or more) had significantly longer times to sternal recumbency than calves born from unassisted calvings or deliveries with slight assistance. Murray (2014) also found that calves born from unassisted calvings had significantly shorter times to sternal recumbency than calves born from calvings that involved dystocia (based on producer calving ease records). Barrier et al. (2013) found that calves born from assisted calvings had a significantly lower transfer of passive immunity (serum IgG) than calves born from unassisted calvings.

Some studies have investigated the influence of mild or severe dystocia on calves, but few have published on the effect of systematic assistance when no signs of calving problems have been detected. A survey conducted in Canada found that $27 \%$ of the 236 dairy producers questioned systematically assisted all of their heifers and cows at calving, regardless of whether they were having difficulty (Villettaz Robichaud et al., 2016). Very little is known about the effect of this practice on the performance of dairy calves. Bellows et al. (1988) and Doornbos et al. (1984) studied the effect of forced early obstetrical assistance (hand pull and calf puller) on beef cows and their calves. They found that this practice had no detrimental effects on the calves' vigor score if correct obstetrical techniques were used. A potential explanation for this has been suggested by Gundelach et al. (2009), who stated that the duration of the second stage of labor seemed to be a key risk factor for perinatal mortality, and Lombard et al. (2007) concluded that relatively simple interventions at calving have the potential to significantly reduce the effect of dystocia on calf mortality and morbidity to $120 \mathrm{~d}$ of age.

The objective of the present study was to investigate the effects of assistance given during the second stage of calving on animals that did not require assistance based on calf presentation and dilation of the dam's cervix and vulva. More specifically, the effects of the intended and actual calving interventions on calves' perinatal mortality, vigor score, time at sternal recumbency, and transfer of passive immunity were examined. The hypothesis was that systematic early assistance at calving, as a management practice, would have no negative effects on the calves if a strict assistance protocol was respected.

\section{MATERIALS AND METHODS}

\section{Animal Enrollment and Data Collection}

The institutional animal care committee of the University of Guelph, monitored by the Canadian Council for Animal Care, approved all procedures described in this study. This study was conducted between October 2011 and May 2012 on a large commercial dairy farm in western Wisconsin (2,300 lactating Holstein cows). Dry cows from 2 milking barns were housed together in a 400-stall transition cow facility during the dry period and for the first 15 to $30 \mathrm{~d}$ after calving. Close-up cows and heifers were housed in 2 freestall pens (90 stalls each) and monitored every 15 to 30 min to detect signs of imminent calving. Animals were moved to an individual maternity pen $(3.7 \times 3.7 \mathrm{~m})$ bedded with fresh straw when any sign of imminent calving was detected (restlessness, bloody vaginal discharge, visible contractions, a visible or broken amniotic sac, or limbs of the emerging calf).

Primiparous and multiparous cows were enrolled in this assistance study at the time of calving only if they met the following specific criteria: (1) they showed signs of imminent calving, unless both front hooves of the calf were visible at the vulva (if previous appearance of both front hooves was in doubt, the cow was automatically excluded); (2) they had no obvious signs of illness, including lameness at the time of calving; and (3) they showed sufficient dilation of the vagina and vulva to permit an unassisted delivery. Dilation was considered sufficient if the forearm of the principal author (MVR) could entirely enter the birth canal alongside the calf. The calf needed to be a live singleton at the time of vaginal assessment and be in a normal anterior presentation for calving. All vaginal examinations were performed by the same trained person (MVR) to ensure consistency. The trained person was present during the entire calving process for each cow enrolled and provided assistance as required, alone or accompanied by farm staff, to ensure consistency in calving management and evaluation of calving ease. Animals that met the criteria and were enrolled in the study were placed in an individual maternity pen and closely observed continuously the calving process.

Once imminent calving was detected, cows were brought to a maternity pen where a vaginal assessment was conducted. After the vaginal assessment, cows and their calves were randomly assigned to 1 of 2 interventions: not assisted (NA) or early assistance (EA). Calving interventions were assigned randomly to cows (second calving or greater) and heifers (first calving) using separate randomization processes, because parity is believed to be an important confounding factor for calving ease (Lombard et al., 2007). Randomization for each parity group was done in blocks of 8 animals to keep numbers in each assigned intervention group balanced, and to avoid any potential bias associated with time of enrollment. The EA intervention was defined as cows receiving assistance during calving (pulling 
the calf) 15 min after the first sight of the calf's 2 front hooves. The NA intervention group had a cutoff time of $1 \mathrm{~h}$ after first sight of both of the calf's front hooves, after which late assistance was provided. The 1-h cutoff was chosen to reduce calf losses for the farm, and to be consistent with the farm's standard operating procedure and recent literature (Schuenemann et al., 2011). Among the animals assigned to the EA intervention, some animals calved very rapidly and were not assisted, because expulsion of the calf was complete before EA was provided. These animals were classified as "too quick to be assisted." Consequently, we had 4 intervention groups: too quick to be assisted (TQ), NA, EA, and late assistance (LA). Because the primary aim of this study was to investigate the effects of assisting all cows at calving, the data were analyzed by assigned interventions (NA and EA) and by actual interventions (TQ, NA, EA, and LA) to allow deeper understanding of the effects of the different calving scenarios.

For vaginal examination and calving assistance, the cow's perineum was first thoroughly cleaned using hot water and betadine $7.5 \%$ surgical scrub (Purdue Products LP, Stamford, CT). Lubricant (Lubiseptic; Boehringer Ingelheim Vetmedica Inc., St. Joseph, MO) was then applied liberally to the obstetrical gloves and the cow's vagina before performing the exam or providing assistance. During vaginal examination, each cow was restrained between the calving pen side and door of the pen. Almost all cows were assisted lying down in their own calving pen. Assistance was provided by pulling calves with a stainless steel chain looped around each front leg, proximal and distal to the fetlock joint (2 loops per leg). Pulling was done using manual force exclusively (as opposed to mechanical force using a fetal extractor). Subjective assessment of calving ease was noted at the end of each calving by the trained individual (MVR), based on the number of people pulling and length and strength of pulling: $1=$ not assisted, 2 = slight assistance (usually 1 person and little force),
$3=$ moderate assistance ( 1 or 2 people and moderate force), and $4=$ strong assistance (usually 2 people with strong force). The number of people pulling to assist a cow during calving was also noted. Time between first appearance of both front hooves and complete expulsion of the calf was recorded as calving duration (min).

In the seconds following complete expulsion of the calf, a vigor score was recorded to assess the calf's viability and vitality. This score was based on meconium staining; responsiveness (head shake, corneal eye reflex, and tongue pinch); tongue and head swelling; heart rate; respiration rate; mucous membrane color; and time to sternal recumbency (min) (Table 1). Heart and respiration rates were recorded in beats/min and breaths/min, respectively. The vigor assessment used for this study was modified from one presented by Murray (2014), where non-vigorous calves have higher total scores; however, it was modified by removing calf movement and tongue length. Time to sternal recumbency was treated as a continuous variable and analyzed separately from the vigor score, with shorter time indicating greater calf vigor.

After calving, calves were separated from their dam before any suckling occurred. Before colostrum feeding, newborn calves were weighed $(\mathrm{kg})$ using an electronic scale (Vet Deck; Brecknell, Fairmont, MN) and identified using a unique ear tag, had their navels dipped using a $7 \%$ tincture of iodine solution, and then were placed in a small group pen $(3.6 \times 3.6 \mathrm{~m})$ bedded with fresh straw for the $24 \mathrm{~h}$ following birth. Other information recorded at that time included dam ID, dam farm of origin, calf ID, sex, birth weight, birth date, birth time, assigned and actual calving intervention groups, and calving ease. Calves' morphometric measures were also taken at this time using a measuring tape: width at the shoulder between the lower tip of the shoulder joints, width between the external tips of the hip bone, and length from the midpoint between the ears to the tail. In the hours following calving, dams' BCS were

Table 1. Vigor score measurements of newborn calves ${ }^{1}$

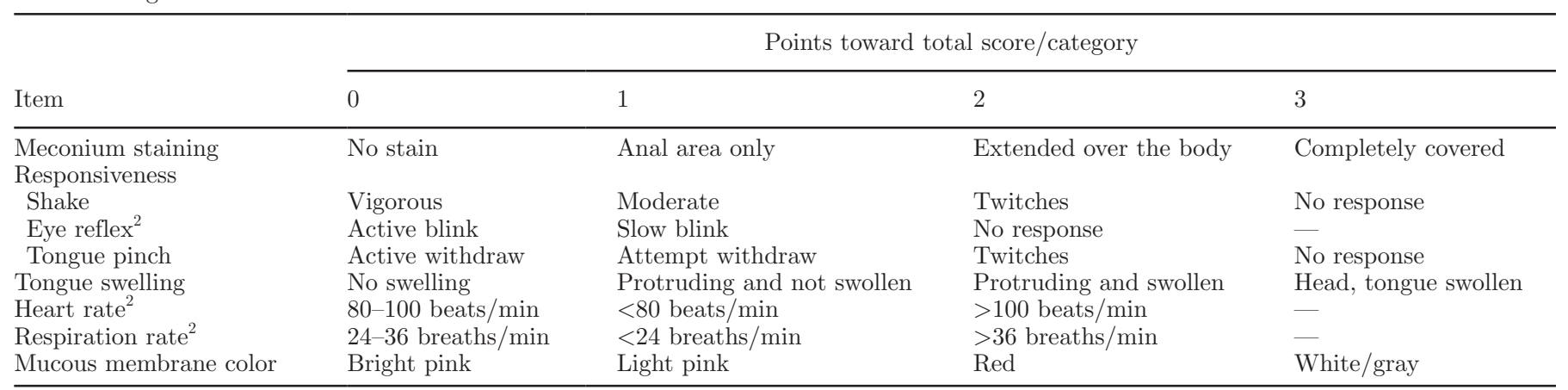

${ }^{1}$ Lower score indicates greater vigor (modified from Murray, 2014).

${ }^{2}$ Scored on a 3 -point scale only. 
assessed using a 5-point scale, their weights were estimated using a girth tape, and their width at the hip bone using a measuring tape (Ferguson et al., 1994).

A 10-mL venous blood sample was collected from each calf using a serum Vacutainer tube (Becton Dickinson, Franklin Lakes, NJ) at birth to assess precolostrum serum IgG concentration. Calves were then fed 1.5 doses (150 g of IgG) of commercially available colostrum replacer (Land O'Lakes Colostrum Replacer; Land O'Lakes Inc., Arden Hills, MN) using an esophageal tube feeder. Calves enrolled in this study were also enrolled in a study looking at the addition of gut-active carbohydrates to the colostrum replacer to enhance transfer of passive immunity. The results of that study showed no significant effect of this intervention on any of the parameters measured here (Villettaz Robichaud et al., 2014). Between colostrum feeding and $24 \mathrm{~h}$ of age, calves were offered $1.9 \mathrm{~L}$ of a commercially available milk replacer twice using a nipple bottle [Amplifier Max. (22\% CP, $20 \%$ crude fat); Land O'Lakes Animal Milk Products, Arden Hills, MN]. At $24 \mathrm{~h}$ of age, a post-colostrum blood sample was taken to assess IgG absorption and calves were moved out of the group pen and into freshly bedded individual hutches outdoors. The definition of stillbirth for this experiment was calves born dead or that died within $24 \mathrm{~h}$ of birth.

\section{Sample Analysis Procedure}

Venous blood samples collected from all calves before (0 to $1 \mathrm{~h}$ ) and after (24) colostrum replacer feeding were refrigerated for a minimum of $6 \mathrm{~h}$ and allowed to clot, after which they were centrifuged (model DSC-158T; Digisystem Laboratory Instruments Inc., New Taipei City, Taiwan) at 12,000 $\times g$ for $10 \mathrm{~min}$, and serum was separated. Serum samples were frozen at $-20^{\circ} \mathrm{C}$ until submission on ice to the Saskatoon Colostrum Co. Ltd. Quality Assurance Laboratory (Saskatoon, SK, Canada) for determination of serum IgG concentration $(\mathrm{mg} / \mathrm{mL})$ using radial immunodiffusion as described by Chelack et al. (1993). We calculated the apparent efficiency of absorption of IgG (AEA, \%) to estimate the percentage of the mass of $\operatorname{IgG}$ consumed that was absorbed by the calf. The equation used was based on the assumption that calf plasma volume is $9.9 \%$ of its birth weight in $\mathrm{kg}$ (Quigley et al., 1998, 2002): [serum IgG concentration at $24 \mathrm{~h}(\mathrm{mg} / \mathrm{mL})$ - serum IgG concentration at $0 \mathrm{~h}(\mathrm{mg} / \mathrm{mL})] \times[$ volume of the plasma of the calf $(\mathrm{L}) / 150 \mathrm{~g}$ of $\operatorname{IgG}$ fed] $\times 100$.

\section{Statistical Analyses}

The data were analyzed using Stata 11 statistical software (StataCorp LP, College Station, TX). We de- termined the a priori sample size for this study based on the proportion of cows with purulent vaginal discharge, assessed 1 month after parturition. This outcome was chosen to calculate the sample size because it was the principal outcome of interest for maternal reproductive health, another aspect of this study to be reported elsewhere. The assumption was that early systematic obstetrical assistance at calving would increase the proportion of cows with purulent vaginal discharge from 17 to $36 \%$, based on the increased odds of purulent vaginal discharge in cows experiencing a difficult calving. Based on the sample size calculation, approximately 125 animals (dams) were needed for each assigned intervention. Data were collected on all calves born from the cows enrolled in the study.

We generated descriptive statistics, including univariable statistics, to describe the characteristics of the calves and their dams at enrollment. For any additional covariate in the models, other than obstetrical intervention, we used an initial cutoff value of $P<0.2$ in the univariable analysis as a prerequisite for inclusion in a multivariable model with calving intervention group. We considered calving ease and the number of people required to pull the calf to be intervening variables between intervention groups and the outcomes measured, and did not include them in subsequent models. Because it was influenced by the assigned interventions, calving duration was considered an intervening variable between intended calving interventions and the outcomes measured, and it was not included in statistical models investigating the effects of assigned interventions. In the actual intervention models, we considered calving duration to be a construction of the actual intervention groups, because duration determined the final intervention category; as a result, duration was not included in subsequent models (Table 2).

We used multivariable linear regression models were used to analyze the effects of the different calving interventions on the following outcomes in calves: vigor score at birth, time to sternal recumbency ( $\mathrm{min}$ ), serum IgG concentration at $24 \mathrm{~h}(\mathrm{mg} / \mathrm{mL})$, and apparent efficiency of absorption of IgG (AEA, \%). We used multivariable logistic regression to analyze the effects of calving interventions on the likelihood of calves being born dead. We then used a manual backward selection model-building strategy to remove all nonsignificant terms, with the exception of calving intervention groups, from the statistical models. Partial F-tests (for linear models), likelihood ratio tests (for logistic models), and Akaike and Bayesian information criteria were used to assess the effect of removing categorical variables from models. The significance level for a variable to be retained in the final models was set at $\alpha=$ 0.05. Intended or actual calving intervention variables 
were forced into each statistical model as they were the variables of interest in this study. The referent calving intervention category for all models was NA. Calf characteristics tested in the models were sex, birth weight, morphometric measures at birth, serum IgG at $0 \mathrm{~h}$, time at vigor score, being licked by the dam at the time vigor score was recorded, and time at colostrum replacer feeding. Cow characteristics tested in the models were parity, farm of origin, BCS, width at hip bone, weight, difference in days between expected and real calving date, and calf/cow weight proportion. Regardless of statistical significance, confounding variables were retained in the models. We identified confounding variables based on the causal diagram, and retained them if their removal resulted in a change of $20 \%$ or greater on the estimated coefficient for calving interventions. Interactions between calving interventions and covariates examined were investigated and kept in the models if they were statistically significant. We did not examine the interaction between actual interventions and parity, because it could not be properly assessed due to the absence of primiparous animals in the TQ group and the limited number of multiparous animals in the LA group.

The assumption of linearity was assessed visually based on the distribution of residuals against the continuous predictors included in the model. If nonlinear, variables were categorized or modeled as a quadratic relationship, if appropriate, based on visual assessment of locally weighted regression curves (LOWESS curves). We identified potential collinearity between predictors using Pearson or Spearman correlations. If 2 variables were highly correlated (rho $\geq|0.8|$ ), only the variable with a higher level of interest to the study was used in the final models.
For linear regression models, we assessed the homoscedasticity of the residuals visually by examining the distribution of the standardized residuals against the predicted values of the outcomes. We assessed the normality of the residuals visually using normal quantile plots. To meet the assumption of normality and homoscedasticity, the outcome time to sternal recumbency was log-transformed. For the linear models, we identified the outliers and influential observations based on visual assessment of the following diagnostics: standardized residuals, leverage, Cook's distance, and Dfits. For the logistics models, we identified the outliers and influential observations based on visual assessment of the following diagnostics: Pearson and deviance residuals, leverage, delta-betas, delta-chi-squared, and delta-deviance. Overall fit of the logistic models was assessed using the Hosmer-Lemeshow goodness-of-fit test.

\section{RESULTS}

\section{Animals and Calving Characteristics}

In total, the 257 calves enrolled in this study (134 heifers and 123 bull calves) were equally distributed between the assigned interventions. They were removed from their dam on average $56 \pm 32$ min (standard deviation, SD) after birth. At birth, on average, calves were $42.0 \mathrm{~kg}$ in weight, $80.9 \mathrm{~cm}$ tall at the hip, and $93.4 \mathrm{~cm}$ long. The mean calf birth weight $(\mathrm{kg})$ for each actual intervention group was (mean $\pm \mathrm{SD}$ ): TQ, 40.7 $\pm 5.5 ; \mathrm{NA}, 42.2 \pm 5.9 ; \mathrm{EA}, 42.4 \pm 5.0$; and LA, 51.5 $\pm 4.2)$. Overall average $( \pm \mathrm{SD})$ dam characteristics were $713 \pm 121 \mathrm{~kg}$ of $\mathrm{BW}, \mathrm{BCS} 3.0 \pm 0.3$, and 69 $\pm 6 \mathrm{~cm}$ width at hip bone. Because of the separate randomization blocks for primiparous and multiparous

Table 2. Distribution of calving ease and calving duration between intended and actual calving intervention groups

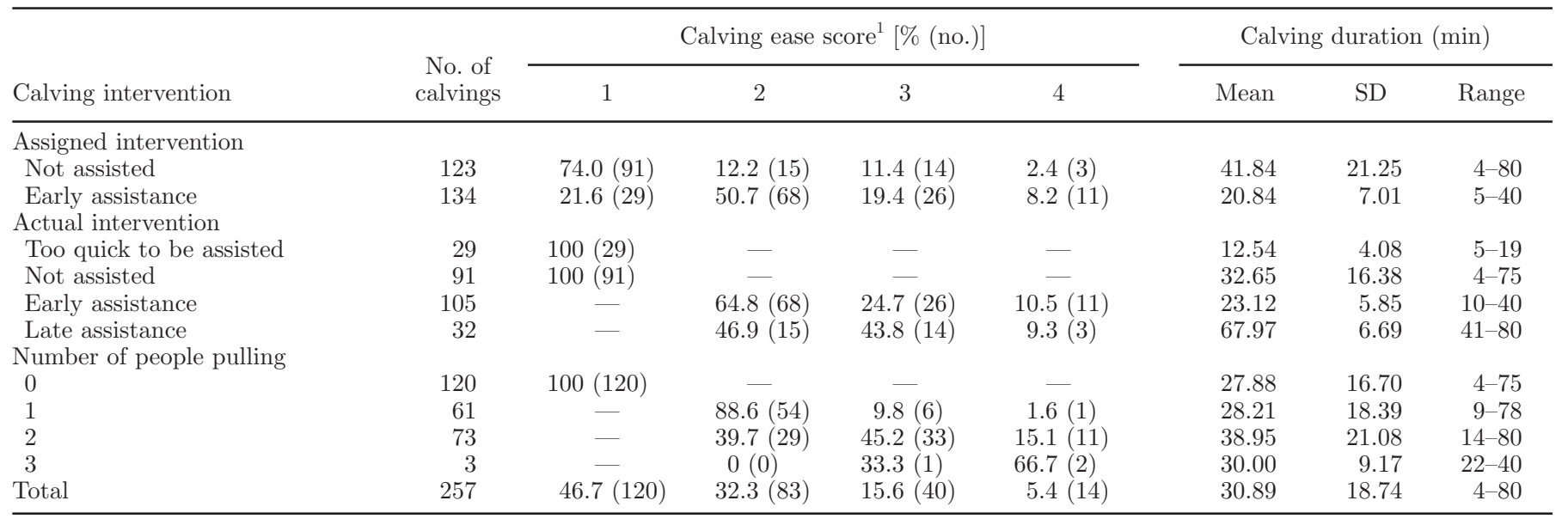

${ }^{1}$ Calving ease noted subjectively as follows: $1=$ not assisted, $2=$ slight assistance, $3=$ moderate assistance, and $4=$ strong assistance. 
cows, dam parity was equally distributed between assigned interventions. We observed a marked difference in the distribution of parity between actual intervention groups, with only multiparous cows (100\%) in the TQ group and a greater proportion of primiparous cows in the LA group (69\% primiparous vs. $31 \%$ multiparous). Specifically, the final groups were 29 multiparous in the TQ group; 21 primiparous and 70 multiparous in the NA group; 41 primiparous and 64 multiparous in the EA group; and 22 primiparous and 10 multiparous in the LA group.

Calving ease, calving duration, and the number of people pulling during calving differed between the assigned and actual calving intervention groups (Table 2 ). In total, $91 \%$ of cows calved lying down. The majority of assisted calvings were assisted by 1 person or 2 people (45 and 53\%, respectively; Table 2). Generally, most assisted calvings (EA and LA) were classified as easy pulls, and the highest proportion (11\%) of hard pulls were in the EA group. Overall, mean calving duration, defined as the time between first sight of both hooves and complete expulsion of the calf, was $31 \mathrm{~min}$ and varied from 4 to $80 \mathrm{~min}$ (Table 2). Average calving duration was longer in the NA assigned intervention and longest in the LA actual intervention group, by definition of the group itself (Table 2).

\section{Effects of Calving Intervention Groups on Stillbirth}

A total of 7 calves ( 5 females and 2 males) were dead when delivered and therefore had no vigor score taken. The overall incidence of stillbirth of the animals enrolled in the study was $2.7 \%$ : $6 \%$ of calves born from primiparous dams and $1 \%$ of calves born from multiparous dams. Of the dead calves, 5 were in the NA and 2 in the EA assigned interventions. By actual intervention, 2 calves were born dead in the NA group, 2 in the EA group, and 3 in the LA group. No calves died between birth and $24 \mathrm{~h}$. Assigned calving intervention did not influence the odds of calves being born dead (Table 3). Among actual interventions, calves born in the LA group were significantly more likely to be stillborn than calves born in the NA group (Table 3) or EA group (odds ratio, 6.9; 95\% CI, 1.01 to $47.50 ; P$ $=0.049$ ). Calf birth weight significantly influenced the likelihood of stillbirth in both the intended and actual calving intervention models. The relationship between calf birth weight and stillbirth was quadratic, with medium-size calves having less chance of being born dead than smaller or larger calves (Figure 1; Table 3). This quadratic relationship led to the inclusion of calf birth weight square in the logistic regression analysis (Table 3).

\section{Effects of Calving Intervention Groups on Indicators of Vigor}

Total vigor score at birth, measured on a continuous scale (0 to 21), varied from 0 to 17 points across the 250 live calves scored, with an overall average of 6.54 points. A lower total vigor score indicates a more vigorous calf. The average time after birth when vigor score was recorded was $53 \mathrm{~s}$, and ranged from 1 to 352 s. Assigned calving interventions, dam parity, time at vigor score, and being licked by the dam at the time of the vigor assessment significantly influenced the vigor score of calves (Table 4). Calves in the assigned EA intervention had significantly lower vigor scores (i.e., were more vigorous) than NA calves (Table 4). Increased time at vigor assessment significantly increased the total vigor score (worse) in the assigned calving intervention model, and calves being licked at the time of the vigor assessment also had lower (better) vigor scores (Table 4). Calves born from multiparous cows

Table 3. Multivariable logistic regression models investigating the effects of assigned and actual calving interventions on the likelihood of calves being born dead

\begin{tabular}{lcccc}
\hline Model, intervention & $\%$ (no./total) & Odds ratio & $95 \%$ CI & $P$-value \\
\hline Model 1, stillbirth & & & & \\
$\quad$ Assigned intervention & $4.1(5 / 123)$ & Referent & - & - \\
$\quad$ Not assisted & $1.5(2 / 134)$ & 0.39 & $0.071,2.117$ & 0.275 \\
Early assistance & - & 0.34 & $0.130,0.870$ & 0.025 \\
Birth weight (kg) & & 1.01 & $1.001,1.023$ & 0.034 \\
Birth weight square & & & \\
Model 2, stillbirth & & & - & - \\
Actual intervention & $0.0(0 / 29)$ & Omitted & - & - \\
$\quad$ Too quick to be assisted & $2.2(2 / 91)$ & Referent & $0.164,13.832$ & 0.718 \\
Not assisted & $1.9(2 / 105)$ & 1.50 & $1.088,97.900$ & 0.042 \\
Early assistance & $9.4(3 / 32)$ & 10.32 & $0.069,0.643$ & 0.006 \\
$\quad$ Late assistance & - & 0.21 & $1.004,1.030$ & 0.008 \\
Birth weight (kg) & - & 1.02 & \\
Birth weight square & &
\end{tabular}

${ }^{1}$ There were no stillborn calves in the "Too quick to be assisted" group. 
Table 4. Multivariable linear regression models investigating the effects of assigned and actual calving interventions on total vigor score in newborn calves ${ }^{1}$

\begin{tabular}{|c|c|c|c|c|c|}
\hline Model, intervention & $\begin{array}{l}\text { No of } \\
\text { calvings }\end{array}$ & Mean (SD, range) & $\beta$ & $95 \% \mathrm{CI}$ & $P$-value \\
\hline \multicolumn{6}{|l|}{ Model 1 , vigor score } \\
\hline Early assistance & 132 & $5.48(3.86,0-16)$ & -2.37 & $-3.317,-1.413$ & $<0.001$ \\
\hline \multicolumn{6}{|l|}{ Parity } \\
\hline Primiparous & 79 & $9.00(4.11,0-17)$ & Referent & - & - \\
\hline \multicolumn{6}{|c|}{ Licked by cow at vigor scoring } \\
\hline No & 233 & $6.69(4.32,0-17)$ & Referent & - & - \\
\hline Yes & 17 & $4.53(2.85,1-9)$ & -2.03 & $-3.922,-0.146$ & 0.035 \\
\hline \multicolumn{6}{|l|}{ Model 2, vigor score } \\
\hline \multicolumn{6}{|l|}{ Actual intervention } \\
\hline Too quick to be assisted & 29 & $3.41(2.72,0-10)$ & -2.97 & $-4.571,-1.364$ & $<0.001$ \\
\hline Not assisted & 89 & $7.02(4.19,0-17)$ & Referent & - & - \\
\hline \multicolumn{6}{|l|}{ Parity } \\
\hline
\end{tabular}

${ }^{1}$ Lower score indicates greater vigor (modified from Murray, 2014).

had significantly lower (better) vigor scores in both assigned and actual calving intervention models (Table 4). Calves in the TQ and EA groups had significantly lower (better) vigor scores than calves in the NA group (Table 4). Calves in the TQ $(\beta,-4.41 ; 95 \% \mathrm{CI},-6.50$ to $-2.32 ; P<0.001)$ and EA $(\beta,-2.92 ; 95 \% \mathrm{CI},-4.52$ to $-1.33 ; P<0.001)$ actual intervention groups also had significantly better vigor at birth than calves in the LA group. Calves in the TQ group had the best (lowest) vigor score of all calves but not significantly different from the EA actual group $(\beta, 1.49 ; 95 \%$ CI, -0.13 to $3.11 ; P=0.071)$. No significant difference was found in calves' vigor score between the NA and LA actual intervention groups (Table 4).

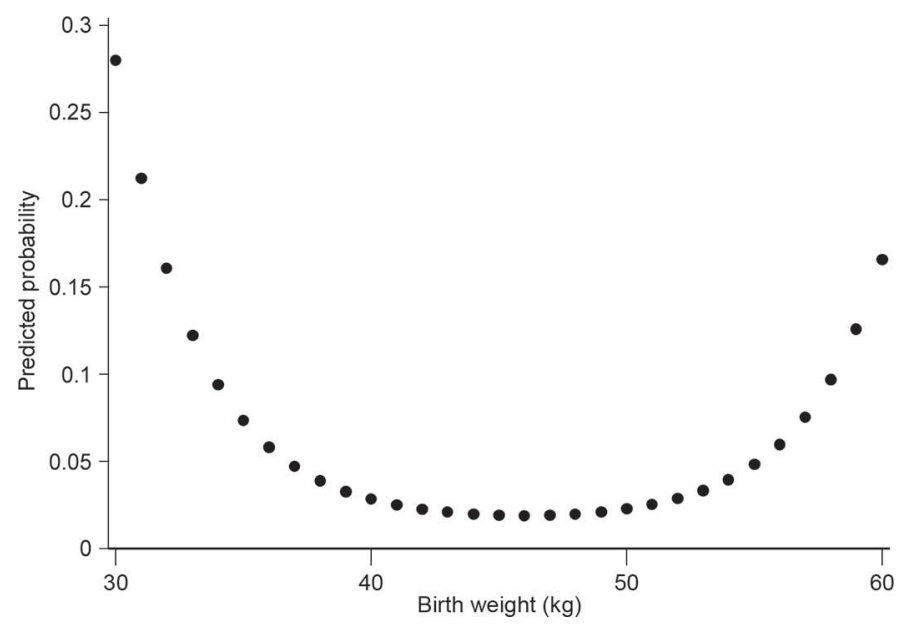

Figure 1. Quadratic relationship between a calf's birth weight and its predicted probability of being stillborn.
The average time to sternal recumbency was $6 \mathrm{~min}$, ranging from 0 to $51 \mathrm{~min}$. Time to sternal recumbency was not influenced by assigned calving intervention (Table 5), but was significantly longer in the EA and the LA groups than in the NA actual intervention group (Table 5). Calves born from multiparous cows achieved sternal recumbency significantly faster than calves born from primiparous dams in both models (Table 5). Dam farm of origin and calf birth weight also significantly influenced the time to sternal recumbency in the assigned interventions model (Table 5). Bigger calves took longer to achieve sternal recumbency.

\section{Effects of Calving Intervention Groups on Transfer of Passive Immunity}

The average time at colostrum replacer feeding was $79 \mathrm{~min}$ and ranged from 25 to $195 \mathrm{~min}$. The great majority of calves $(89 \%)$ received their colostrum replacer within the $2 \mathrm{~h}$ advised timeframe (Godden, 2008). Serum IgG concentrations at $0 \mathrm{~h}$ averaged $0.4 \mathrm{mg} /$ $\mathrm{mL}$, ranging from 0.2 to $2.4 \mathrm{mg} / \mathrm{mL}$. These were in the normal range identified in other studies (Godden et al., 2009a). Mean IgG at $24 \mathrm{~h}$ for all calves was $20.4 \mathrm{mg} /$ $\mathrm{mL}$, ranging from 8.4 to 33.5 . Overall, only 2 calves of the 233 calves tested $(0.9 \%)$ did not achieve adequate transfer of passive immunity, set at serum $\operatorname{IgG}>10$ $\mathrm{mg} / \mathrm{mL}$ (Godden, 2008; Beam et al., 2009). The AEA of serum IgG absorption was $54.8 \%$, ranging from 20.7 to $93.3 \%$. Serum IgG at $24 \mathrm{~h}$ was significantly affected by calving intervention group, both assigned and actual, in interaction with calves' birth weights (Table 6). 
Table 5. Multivariable linear regression models investigating the effects of assigned and actual calving interventions on log-transformed time to sternal recumbency in newborn calves

\begin{tabular}{|c|c|c|c|c|c|}
\hline Model, intervention & $\begin{array}{l}\text { No. of } \\
\text { calvings }\end{array}$ & Mean $(\mathrm{SD}, \text { range })^{1}$ & $\beta$ & $95 \% \mathrm{CI}$ & $P$-value \\
\hline \multicolumn{6}{|c|}{ Model 1, log (time to sternal recumbency) (min) } \\
\hline Not assisted & 118 & $6.43(7.75,0-44)$ & Referent & - & - \\
\hline Early assistance & 132 & $5.92(6.12,0-51)$ & 0.16 & $-0.059,0.387$ & 0.149 \\
\hline \multicolumn{6}{|l|}{ Parity } \\
\hline Primiparous & 79 & $9.10(9.93,0-51)$ & Referent & - & - \\
\hline \multicolumn{6}{|l|}{ Dam farm of origin } \\
\hline Dairy 1 & 117 & $5.19(5.82,0-33)$ & Referent & - & - \\
\hline Dairy 2 & 133 & $7.01(7.69,0-51)$ & 0.24 & $0.021,0.468$ & 0.032 \\
\hline Birth weight $(\mathrm{kg})$ & & - & 0.03 & $0.005,0.051$ & 0.016 \\
\hline \multicolumn{6}{|c|}{ Model 2, log (time to sternal recumbency) (min) } \\
\hline \multicolumn{6}{|c|}{ Actual intervention } \\
\hline Too quick to be assisted & 29 & $3.67(3.25,0-17)$ & -0.08 & $-0.457,0.289$ & 0.658 \\
\hline Not assisted & 89 & $4.96(6.02,0-33)$ & Referent & - & - \\
\hline
\end{tabular}

${ }^{1}$ Time to sternal recumbency before log-transformation (min).

Overall, smaller calves had higher serum IgG at $24 \mathrm{~h}$ than bigger calves. The decrease in serum IgG at $24 \mathrm{~h}$ with increasing birth weight was more pronounced in calves born from assigned NA calvings than assigned EA calvings (Figure 2a). Again, IgG levels at $24 \mathrm{~h}$ declined with increasing calf size, but the intercept and slope of the predicted value varied with actual interventions (Figure 2b). Calves born from multiparous cows had significantly lower serum IgG at $24 \mathrm{~h}$, but increasing time between birth and colostrum replacer feeding slightly increased it (Table 6). Calving interventions did not affect AEA, but AEA increased with higher calf/cow weight proportion (Table 6). In the assigned calving interventions model, time between birth and colostrum replacer feeding positively influenced AEA, and male calves had significantly higher AEA (Table $6)$. In the actual calving intervention model, the difference between actual and expected calving date was a confounding variable for AEA and needed to be controlled for in the model (Table 6).

\section{DISCUSSION}

Early assistance provided during calving as a general management practice to cows that did not present with obvious characteristics of dystocia did not negatively affect their calves in terms of stillbirth, vigor indicators, or transfer of passive immunity. Early assistance actually improved calf vigor at birth compared with no assistance at calving and reduced the incidence of stillbirth compared with providing late assistance.
Most published research has focused on the effects of calving difficulties on calves, but very little work has examined the effect of assistance itself, without signs of calving problems, which is a common practice on many farms. In the present study, we used block randomization to ensure that an equal proportion of heifers and cows received the assigned interventions, because parity is the most important factor influencing calving ease (Philipsson, 1976; Gevrekci et al., 2011). Calf birth weight and maternal pelvic size are the 2 factors of importance in feto-pelvic disproportion, the most common type of dystocia in dairy cattle (Johanson and Berger, 2003; Mee, 2008a). Overall, the characteristics of the calves and dams were similarly distributed between assigned interventions. Some differences were found among the actual interventions, mainly in the LA group, which had the largest calves and a higher proportion of primiparous dams, but this was expected, as it is consistent with findings in the current literature.

The overall stillbirth rate for this study was $2.7 \%$, much lower than recently reported stillbirth rates of $7.2 \%$ for singletons on commercial farms (Silva del Río et al., 2007; USDA, 2009). The stillbirth rates in the present study for primiparous $(6 \%)$ and multiparous (1\%) dams were even lower than the objective set for welfare concern of $3 \%$ in multiparous and $8 \%$ in primiparous dams (Fishwick, 2011). The odds of stillbirth were not different between assigned calving interventions but when considering actual interventions, the LA group had more stillbirths than the NA and EA groups. Perinatal mortality has several causes, but one 
Table 6. Multivariable linear regression models investigating the effects of assigned and actual calving interventions on serum IgG and apparent efficiency of absorption at $24 \mathrm{~h}$ in newborn calves

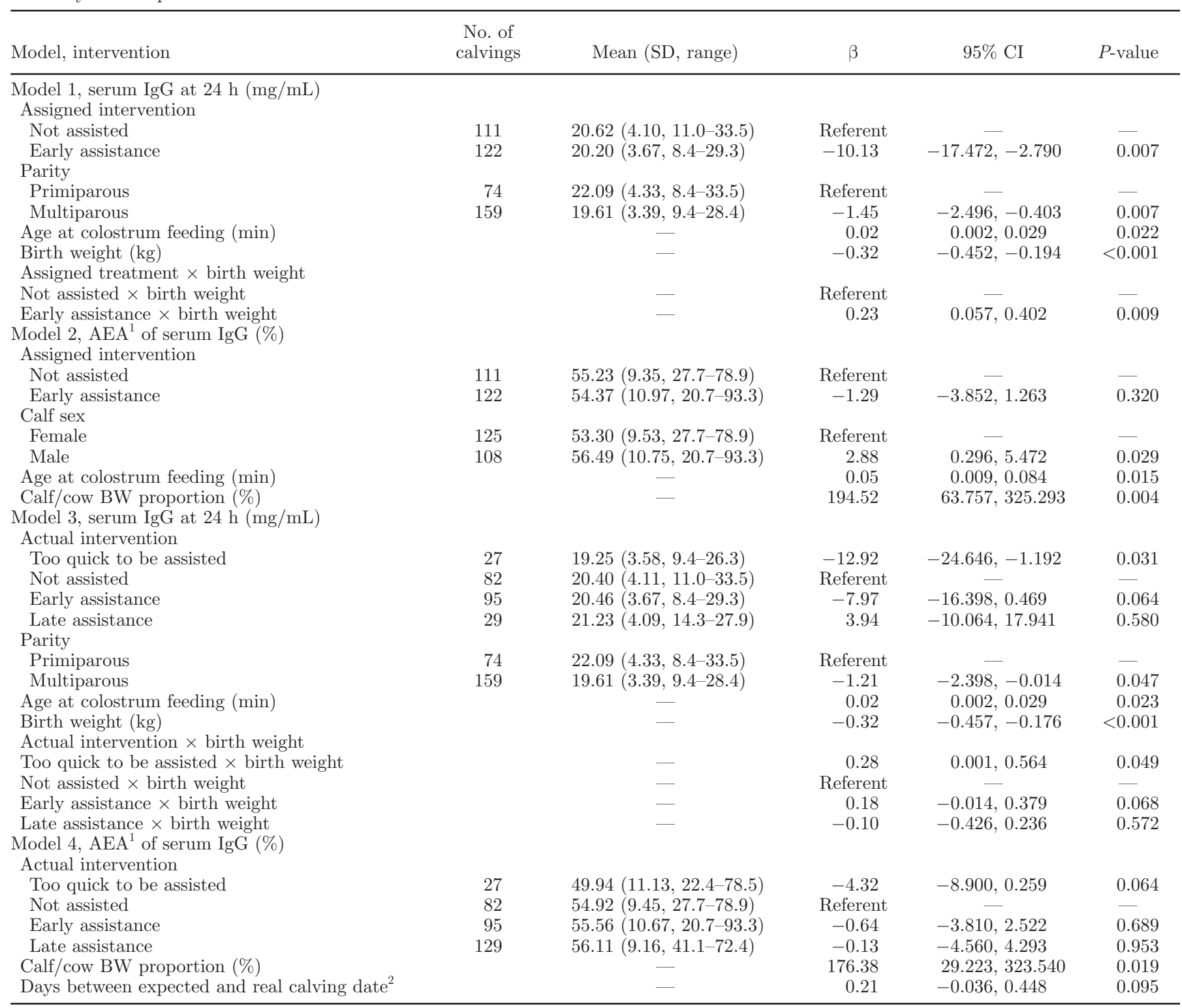

\footnotetext{
${ }^{1}$ Apparent efficiency of absorption of serum IgG at $24 \mathrm{~h}$.

${ }^{2}$ Actual calving date minus expected calving date.
}

of the major ones is disproportion between calf weight and dam pelvic bone size (Mee, 2008a). The significance of calf birth weight as a predictor of stillbirth in the intended and actual calving interventions was not surprising; it has been found to account for $50 \%$ of phenotypic variance in dystocia cases (Philipsson, 1976; Meijering, 1984). In the present study, we found a quadratic relationship between the odds of stillbirth and calf birth weight. Meijering (1984) previously described the existence of a nonlinear relationship between calf birth weight and the incidence of dystocia and stillbirth, having both higher and lower threshold values, leading to an optimum range of birth weights for optimal calf viability. They found that smaller calves were more likely to be born from primiparous dams, and that these dams were more likely to experience dystocia and have stillborn calves (Meijering, 1984). Johanson and Berger (2003) defined the relationship they found between birth weight and perinatal mortality with a threshold of $42 \mathrm{~kg}$. Based on the results of our study, the optimal birth weight of Holstein calves to reduce the odds of stillbirth would be around $45 \mathrm{~kg}$ (Figure 1).

The duration of the second stage of labor is also a key risk factor for perinatal mortality, and reference times 

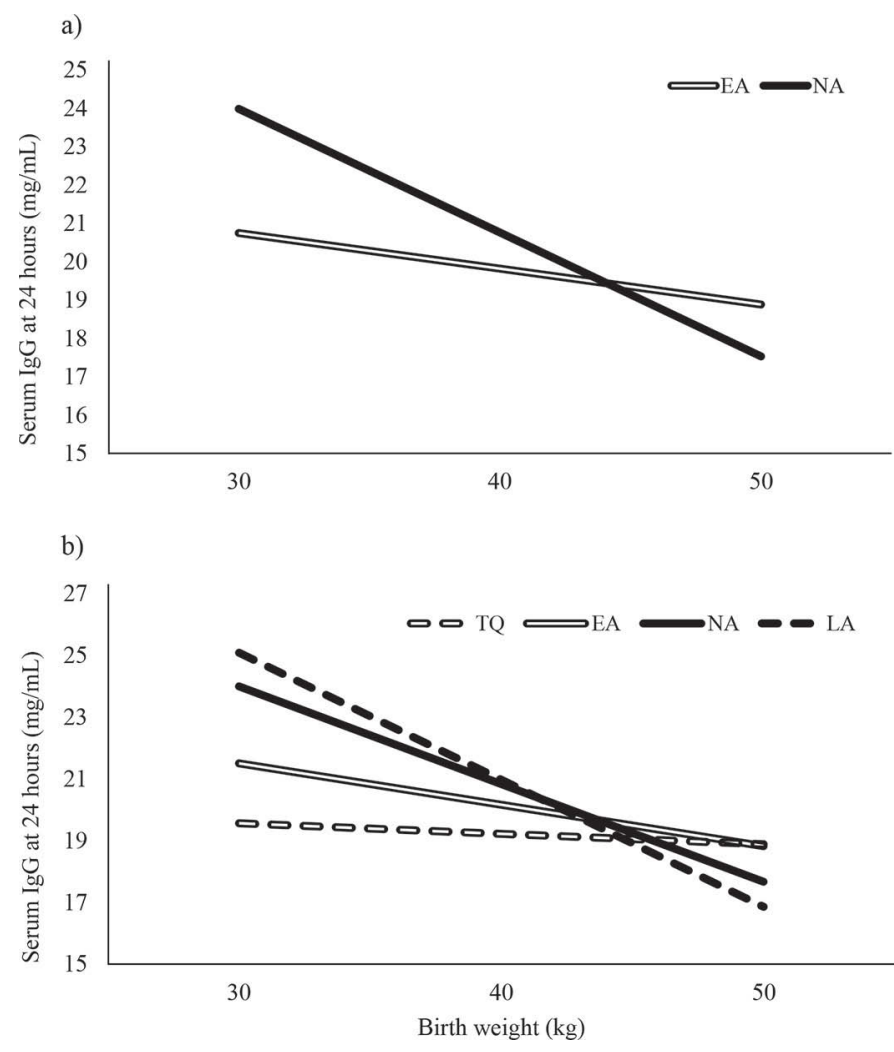

Figure 2. Effect of the interaction between (a) assigned $(\mathrm{EA}=$ early assistance; $\mathrm{NA}=$ not assisted) and (b) actual calving interventions $(\mathrm{TQ}=$ too quick to be assisted; $\mathrm{EA}=$ early assistance; $\mathrm{NA}=$ not assisted; LA = late assistance) and calves' birth weight on serum IgG concentration at $24 \mathrm{~h}$ of age.

for assistance to limit calf losses have been proposed (Gundelach et al., 2009). When the second stage of labor is less than $2 \mathrm{~h}$, the odds of perinatal mortality are significantly lower than in longer calvings, and 65 min after hoof appearance has been suggested as a threshold for obstetric intervention (Gundelach et al., 2009; Schuenemann et al., 2011). As per definition, the actual LA group was given assistance $1 \mathrm{~h}$ after the first sight of both of the calves' front hooves, resulting in a much longer duration of the second stage of labor than the other actual intervention groups. The results of the present study suggest that a cutoff of $60 \mathrm{~min}$ still leads to a higher likelihood of calf mortality than early obstetrical assistance $15 \mathrm{~min}$ after the appearance of a calf's feet. Lombard et al. (2007) suggested that relatively simple interventions have the potential to significantly reduce the effect of dystocia on calf mortality, and the early assistance provided to cows in this study achieved reduced mortality compared with the LA group.

In the present study, calves assigned to the EA group had better vigor than calves assigned to the NA group. Among actual calving interventions, TQ calves had the best vigor scores, and LA calves had the worst scores, suggesting that calving duration has more effect on calf vigor than assistance itself. In the literature, calves requiring assistance during calving have significantly reduced vigor compared with calves born from unassisted calvings (Barrier et al., 2012; Murray, 2014). Comparable to the present study, previous research has also found that calves born from multiparous cows have better vigor than calves born from primiparous cows (Doornbos et al., 1984). Moreover, calves born from hard pulls had a greater risk of reduced vigor than calves born from easy pulls (Murray, 2014). Calves with worse vigor at birth are also more acidotic, with higher blood partial pressure of $\mathrm{CO}_{2}$ than calves with better vigor scores, possibly reflecting insufficient blood oxygenation during calving (Bleul et al., 2007; Murray, 2014). The differences in vigor scores found in the present study among the actual interventions may be because calves experienced a longer period with insufficient blood oxygenation as a result of longer calving durations in the LA and NA groups than in the TQ and EA groups.

Calves took longer to achieve sternal recumbency after assisted calving (EA and LA intervention groups) than after unassisted calving (TQ and NA intervention groups). Time to sternal recumbency is a valuable, practical, objective, and reliable diagnostic tool for evaluating the viability of calves. Schuijt and Taverne (1994) established that calves attaining sternal recumbency 16 min or more after birth were significantly more likely to be nonviable. Less than $10 \%$ of the calves in the present study took longer than 16 min to achieve sternal recumbency. Similar to the present research, previous research has also found that calves delivered with forced extraction needed a significantly longer time to achieve sternal recumbency than those with unassisted birth (Schuijt and Taverne, 1994; Murray, 2014). Previous studies have found that calves may experience subcutaneous bleeding, dislocations, fractures, and other bone, muscle, and soft tissue trauma during delivery, especially when excessive force is used during traction (Winstanley, 1973; Schuijt, 1990; Berglund et al., 2003; Aksoy et al., 2009). The difference in sternal recumbency found in this study may be an indication of potential discomfort or damage to the calves' muscles (especially to the front limbs), or other health issues. For instance, calves that experience difficult or prolonged calving also have a higher incidence of hypoxia and acidosis than calves from eutotic births, and they take longer to recover from these conditions (Murray, 2014; Schuijt and Taverne, 1994). Hypoxia and acidosis could explain the increased time to sternal recumbency observed following assisted interventions in this study (Mee, 2008b; Murray and Leslie, 2013). Part of 
the delay to achieving sternal recumbency in assisted calves could also be explained by the body position of the calf once it has been completely expelled. When assistance is provided, the calf usually has all limbs extended to their maximum at the time of expulsion; during naturally occurring calving, calves may partially retract their front limbs toward their abdomen before expulsion of their hind limbs is completed, so that they are in a position closer to sternal recumbency at expulsion than assisted calves.

Immunoglobulin $\mathrm{G}$ at $24 \mathrm{~h}$ was significantly affected by an interaction between calving intervention and calf birth weight. The effects of calving ease on the transfer of passive immunity have been previously examined, with contradictory findings. Barrier and al. (2013) reported significantly lower transfer of passive immunity in calves born from farmer-assisted calvings than in calves born from unassisted calvings, based on the results of zinc sulfate turbidity tests. In contrast, other studies have found no evidence that calving ease affected serum IgG, IgM, or IgA at $24 \mathrm{~h}$ (Stott and Reinhard, 1978), or the proportion of calves experiencing failed transfer of passive immunity (Beam et al., 2009). In the present study, the AEA was not different between intervention groups but was generally higher than expected, with AEA of IgG reported in the literature ranging from 36 to $51 \%$ using the same colostrum replacer product (Godden et al., 2009a,b). Because an equal dose of colostrum replacer was used for each calf and fed using an esophageal tube, all calves received the same quantity $(150 \mathrm{~g})$ and quality of colostrum replacer. Because bigger calves have a greater blood volume (Quigley et al., 1998), a dilution effect resulted in lowering the concentration of $\mathrm{IgG} / \mathrm{mL}$ of serum at 24 $\mathrm{h}$ for bigger calves, explaining the association between birth weight and both serum IgG at $24 \mathrm{~h}$ and AEA of serum IgG at $24 \mathrm{~h}$.

The differences in the distribution of animals between actual intervention groups resulted in a small number of animals in the TQ and LA groups. For some of the outcomes, the lack of statistically significant differences between intervention groups even when numerical differences were found may have been a result of these small numbers. Another potential limitation of this study was the very controlled environment in which the calving interventions were applied. The use of a single observer for all calvings and for assistance strengthened the study by limiting the potential bias introduced by variation in observers or obstetricians, but also reduced the external validity, because this variation will affect the overall performance achieved by this intervention within farms. Investigating the specific effects of calving duration, force used during assistance, and duration of assistance could help explain some of the significant differences found between actual calving interventions. Based on our results, this study demonstrates that early assistance at calving, when the dam does not present signs of calving difficulty, can be done without causing harm and with some benefits to the calves. The effects of this management practice on the health outcomes of replacement heifers and the dams still need to be evaluated before formal recommendations can be made about this practice. Information on the effects of early assistance on the dams (health, reproduction, survival) was collected during the present study and will be presented in future publications. A multi-farm study would also provide insight into how much variation in these intervention effects is influenced by farm-level factors.

\section{CONCLUSIONS}

Giving early, hygienic assistance during calving to cows that do not present with obvious signs of dystocia as a general management practice, did not negatively affect their calves. Early assistance improved calf vigor and reduced the likelihood of stillbirth compared with late assistance. It is important to note that the present study investigated systematic early obstetrical assistance at calving as a management practice on the dairy calves' performance in early life and not the effect of dystocia itself. It has been previously suggested that relatively simple interventions during calving can significantly reduce the effect of dystocia on calf mortality and morbidity (Lombard et al., 2007). The early intervention method used in this experiment, including high cleanliness and only human force during pulling, did not have negative effects, and its adoption may improve calf health by reducing the number of lateassisted calvings. Further research is needed to investigate the effect on cows of early assistance at calving as a management practice and the potential benefits or risks of such practices on other management issues on dairy farms.

\section{ACKNOWLEDGMENTS}

Financial support was provided by Valacta Inc. (Montréal, QC, Canada), the Natural Sciences and Engineering Research Council (Ottawa, ON, Canada), the Ontario Veterinary College (Guelph, ON, Canada) and the Ontario Ministry of Agriculture, Food and Rural Affairs (Guelph, ON, Canada). The authors also thank Jeffrey Rushen (University of British Columbia, Agassiz, BC, Canada) for his help with the revision of the manuscript, Ken Leslie (University of Guelph, Guelph, ON, Canada) for his support with the planning of this experiment, and the owners (John Vrieze and Brittany Vrieze) and staff (Rolando Juarez, Antonio Oncha, 
Alejo Gonzalez, Anselmo Ayohua, Nicolas Quechulpa, Juan Quechulpa, Rafael Orta, Pedro Galvan and Jacob Flores) at Emerald Dairy II/Calf-A (Emerald, WI). Without their constant assistance and support, the completion of this study would have been impossible.

\section{REFERENCES}

Aksoy, O., I. Ozaydin, E. Kilic, S. Ozturk, E. Gungor, B. Kurt, and H. Oral. 2009. Evaluation of fractures in calves due to forced extraction during dystocia: 27 cases (2003-2008). Kafkas Univ. Vet. Fak. Derg. 15:339-344.

Arnott, G., D. Roberts, J. A. Rooke, S. P. Turner, A. B. Lawrence, and K. M. D. Rutherford. 2012. Board invited review: The importance of the gestation period for welfare of calves: Maternal stressors and difficult births. J. Anim. Sci. 90:5021-5034.

Barrier, A. C., M. J. Haskell, S. Birch, A. Bagnall, D. J. Bell, J. Dickinson, A. I. Macrae, and C. M. Dwyer. 2013. The impact of dystocia on dairy calf health, welfare, performance and survival. Vet. J. 195:86-90.

Barrier, A. C., E. Ruelle, M. J. Haskell, and C. M. Dwyer. 2012. Effect of difficult calving on the vigour of the calf, the onset of maternal ehavior, and some behavioural indicators of pain in the dam. Prev. Vet. Med. 103:248-256.

Beam, A. L., J. E. Lombard, C. A. Kopral, L. P. Garber, A. L. Winter, J. A. Hicks, and J. L. Schlater. 2009. Prevalence of failure of passive transfer of immunity in newborn heifer calves and associated management practices on US dairy operations. J. Dairy Sci. 92:3973-3980.

Bellows, R. A., R. E. Short, R. B. Staigmiller, and W. L. Milmine 1988. Effects of induced parturition and early obstetrical assistance in beef cattle. J. Anim. Sci. 66:1073-1080.

Berglund, B., L. Steinbock, and M. Elvander. 2003. Causes of stillbirth and time of death in Swedish Holstein calves examined post mortem. Acta Vet. Scand. 44:111-120.

Bleul, U., B. Lejeune, S. Schwantag, and W. Kahn. 2007. Blood gas and acid-base analysis of arterial blood in 57 newborn calves. Vet. Rec. 161:688-691.

Chelack, B. J., P. S. Morley, and D. M. Haines. 1993. Evaluation of methods for dehydration of bovine colostrum for total replacement of normal colostrums in calves. Can. Vet. J. 34:407-412.

Doornbos, D. E., R. A. Bellows, P. J. Burfening, and B. W. Knapp. 1984. Effects of dam age, prepartum nutrition and duration of labor on productivity and postpartum reproduction in beef females. J. Anim. Sci. 59:1-10.

Ferguson, J. D., D. T. Galligan, and N. Thomsen. 1994. Principal descriptors of body condition score in Holstein cows. J. Dairy Sci. 77:2695-2703.

Fishwick, J. 2011. Welfare issues associated with calving. Cattle Pract. 19:1-2.

Gevrekci, Y., Y. Akbas, and K. Kizilkaya. 2011. Comparison of different models in genetic analysis of dystocia. Kafkas Univ. Vet. Fak. Derg. 17:387-392.

Godden, S. 2008. Colostrum management for dairy calves. Vet. Clin. North Am. Food Anim. Pract. 24:19-39.

Godden, S. M., D. M. Haines, and D. Hagman. 2009a. Improving passive transfer of immunoglobulins in calves I: Dose effect of feeding a commercial colostrum replacer. J. Dairy Sci. 92:1750-1757.

Godden, S. M., D. M. Haines, K. Konkol, and J. Peterson. 2009b. Improving passive transfer of immunoglobulins in calves. II: Interaction between feeding method and volume of colostrum fed. J. Dairy Sci. 92:1758-1764.

Gundelach, Y., K. Essmeyer, M. K. Teltscher, and M. Hoedemaker. 2009. Risk factors for perinatal mortality in dairy cattle: Cow and foetal factors, calving process. Theriogenology 71:901-909.
Johanson, J. M., and P. J. Berger. 2003. Birth weight as a predictor of calving ease and perinatal mortality in Holstein cattle. J. Dairy Sci. 86:3745-3755.

Lombard, J. E., F. B. Garry, S. M. Tomlinson, and L. P. Garber. 2007. Impacts of dystocia on health and survival of dairy calves. J. Dairy Sci. 90:1751-1760.

Mee, J. F. 2008a. Prevalence and risk factors for dystocia in dairy cattle: A review. Vet. J. 176:93-101.

Mee, J. F. 2008b. Newborn dairy calf management. Vet. Clin. North Am. Food Anim. Pract. 24:1-17.

Meijering, A. 1984. Dystocia and stillbirth in cattle-A review of causes, relations and implications. Livest. Prod. Sci. 11:143-177.

Murray, C. 2014. Characteristics, risk factors and management programs for vitality of newborn dairy calves. $\mathrm{PhD}$ Thesis. Univ. of Guelph, Guelph, ON, Canada.

Murray, C. F., and K. E. Leslie. 2013. Newborn calf vitality: Risk factors, characteristics, assessment, resulting outcomes and strategies for improvement. Vet. J. 198:322-328.

Philipsson, J. 1976. Calving performance and calf mortality. Livest. Prod. Sci. 3:319-331.

Quigley, J. D., J. J. Drewry, and K. R. Martin. 1998. Estimation of plasma volume in Holstein and Jersey calves. J. Dairy Sci. 81:1308-1312.

Quigley, J. D., C. J. Kost, and T. M. Wolfe. 2002. Absorption of protein and IgG in calves fed a colostrum supplement or replacer. J. Dairy Sci. 85:1243-1248.

Schuenemann, G. M., I. Nieto, S. Bas, K. N. Galvao, and J. Workman. 2011. Assessment of calving progress and reference times for obstetric intervention during dystocia in Holstein dairy cows. J. Dairy Sci. 94:5494-5501.

Schuijt, G. 1990. Iatrogenic fractures of ribs and vertebrae during delivery in perinatally dying calves: 235 cases (1978-1988). J. Am. Vet. Med. Assoc. 197:1196-1202.

Schuijt, G., and M. A. M. Taverne. 1994. The interval between birth and sternal recumbency as an objective measure of the vitality of newborn calves. Vet. Rec. 135:111-115.

Silva del Río, N., S. Stewart, P. Rapnicki, Y. M. Chang, and P. M. Fricke. 2007. An observational analysis of twin births, calf sex ratio, and calf mortality in Holstein dairy cattle. J. Dairy Sci. 90:1255-1264.

Spicer, H. M., L. A. Goonewardene, A. O. McNeill, and W. L. Slack. 1994. Alberta dairy farm survey response. J. Dairy Sci. 77:34603472 .

Stott, G. H., and E. J. Reinhard. 1978. Adrenal function and passive immunity in the dystocial calf. J. Dairy Sci. 61:1457-1461.

Tenhagen, B. A., A. Helmbold, and W. Heuwieser. 2007. Effect of various degrees of dystocia in dairy cattle on calf viability, milk production, fertility and culling. J. Vet. Med. A Physiol. Pathol. Clin. Med. 54:98-102.

USDA. 2009. Dairy 2007: Part IV: Reference of dairy cattle health and management practices in the United States, 2007. No. N494.0209. Centers for Epidemiology and Animal Health, USDA-APHIS-VS, Fort Collins, CO.

Villettaz Robichaud, M., A. M. de Passillé, D. L. Pearl, S. J. LeBlanc, S. M. Godden, D. Pellerin, E. Vasseur, J. Rushen, and D. B. Haley. 2016. Calving management practices on Canadian dairy farms: Prevalence of practices. J. Dairy Sci. 99:2391-2404.

Villettaz Robichaud, M., S. M. Godden, D. H. Haines, D. B. Haley, and D. L. Pearl. 2014. Addition of gut active carbohydrates to colostrum replacer does not improve passive transfer of immunoglobulin G in Holstein dairy calves. J. Dairy Sci. 97:5700-5708.

Winstanley, E. W. 1973. Fractures of the fore-leg caused by traction at calving. Ir. Vet. J. 27:218-221. 